\title{
Identical Location Aberration Corrected TEM Study on the Degradation Mechanism of Platinum Nanoparticles on Carbon Nanotubes in High Temperature Fuel Cells
}

\author{
Somaye Rasouli ${ }^{1}$, Mohamed R Berber ${ }^{2}$, Inas H Hafez ${ }^{2}$, Tsuyohiko Fujigaya ${ }^{2}$, Paulo Ferreira ${ }^{1}$, \\ Naotoshi Nakashima ${ }^{2}$ \\ 1. Materials Science and Engineering Program, University of Texas at Austin, Austin TX 78712, \\ USA \\ 2. Department of Applied Chemistry, Kyushu University, Fukuoka 819, Japan
}

The low durability of PEMFCs remains a key challenge in fuel cell commercialization. In this regard, carbon nanotubes are recognized as a promising alternative supporting material for fuel cell electrocatalysts due to their higher electrical conductivities and higher durability, when compared to carbon black. In fact, using Pt nanoparticles supported on carbon nanotubes (CNTs) wrapped in poly (vinylphosphonic acid)-doped polybenzimidazole (PVPA-PBI) makes a remarkably high durable fuel cell, thus opening the door for the next generation of these devices [1].

In this work, the degradation mechanism of $3.4 \mathrm{~nm}$ Pt nanoparticle catalysts supported on PVPA-PBI wrapped CNTs was investigated by aberration corrected TEM, before and after voltage cycling. In order to carry this experiment, Pt/PVPA-PBI/CNT powder was deposited on a gold grid attached to a gold plate, which was used as a working electrode in a three electrode electrochemical cell (Fig. 1). To simulate the effect of fuel cell cycling, the TEM grid was cycled between 0.7 and $1.2 \mathrm{~V}$ in $\mathrm{N}_{2}$ saturated $0.1 \mathrm{HClO}_{4}$ liquid electrolyte. In this fashion, pre-defined locations of the electrocatalyst on the TEM grid were analyzed before and after cycling by an aberration-corrected JEOL ARM 200F.

The main mechanism for the loss of electrochemical active surface area was found to be particle motion, followed by coalescence. In contrast to the cases where Pt is supported on carbon black and Nafion ionomer, no particle dissolution and/or re-deposition (Ostwald ripening) is found after voltage cycling. This is confirmed by comparing the size of the spherical particles in the cathode of the MEA before and after 10,000 cycles, which shows no significant growth. On the other hand, the number of coalesced particles has increased considerably after voltage cycling (Fig. 2).

The coalescence mechanism of the nanoparticles is illustrated in Fig. 3, which shows the same location for the electrocatalyst, before and after voltage cycling. In particular, Fig. 3a shows two well separated nanoparticles on the left side of the CNT (square 1), which move until they contact each other and coalesce via neck growth (Fig. 3b). On the other side of the CNT (square 2 in Fig.3a and $b$ ), the change in the orientation of an individual nanoparticle can be observed after voltage cycling. As a result, a larger fraction of the nanoparticle surface area has been covered by carbon and will thus be inaccessible to the fuel. Fig 3c shows six individual particles (square 1) close to each other with a different orientation (Fast Fourier Transform shown in the inset of Fig. 3.c). During voltage cycling, the particles move toward each other and make contact. In order to lower the total energy of the grain boundaries, particle rotation or orientation alignment of the planes at the interface of the particles leads to formation of a larger single crystal particle (FTT in the inset of Fig. 3d). Expansion of the ionomer phase and severe structural deformation of the carbon nanotube is another source of degradation of the electrocatalyst which is shown in Fig 3c,d and e,f. 


\section{References:}

[1] M Berber, T Fujigaya, N Nakashima, Chemcatchem, 6 (2014), p. 567.

[2] F Perez-Alonsoa, CF Elkjær, SS Shim, BL Abrams, IEL Stephens, I Chorkendorff, Journal of Power Sources 196 (2011), p. 6085.

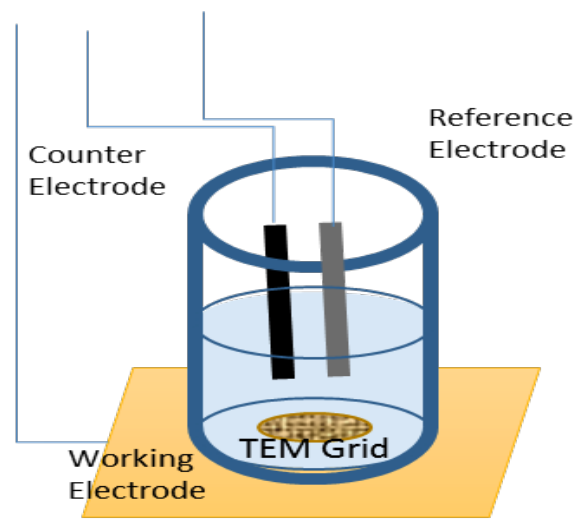

Figure 1. Schematic of the three electrode electrochemical cell with Au TEM grid as working electrode

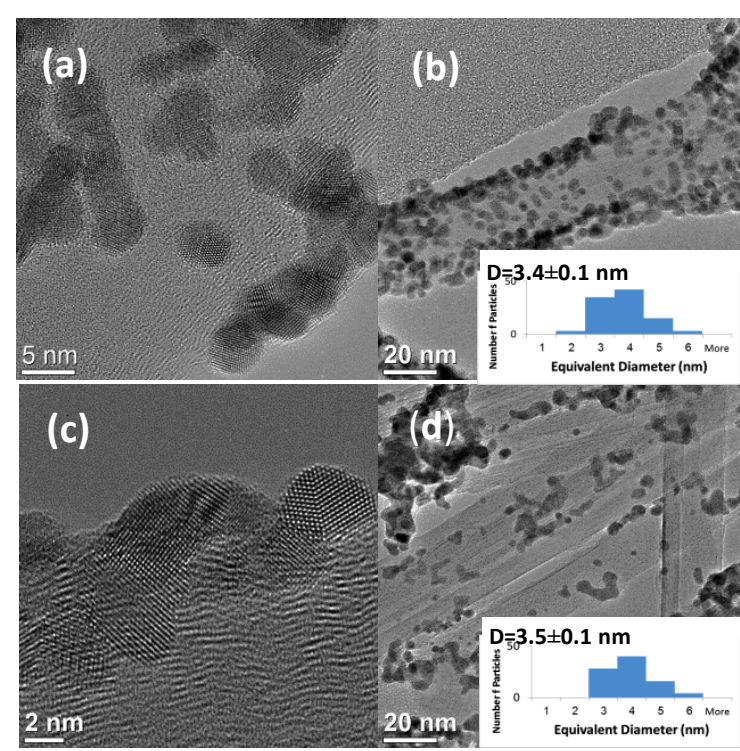

Figure 2. (a) and (b) TEM images of thec Pt/PVPA-PBI/CNT powder, (c) and (d) cathode of the MEA after 10000 voltage cycling with Particle size distribution of the spherical particles in the inset

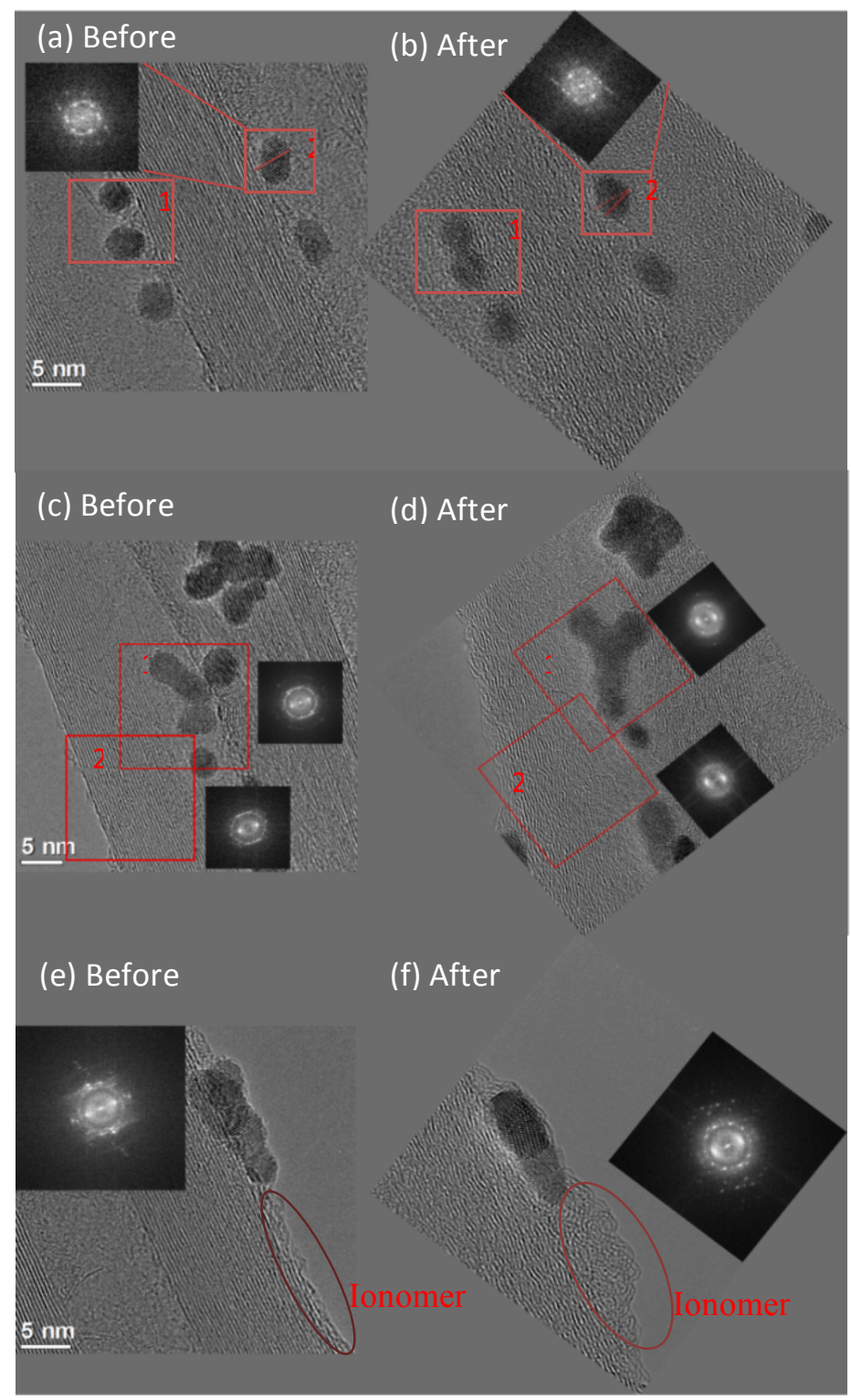

Figure 3 Aberration corrected TEM images of the Pt nanoparticles on PVPA-PBI wrapped carbon nanotubes before and after 1000 voltage cycling 